\title{
Diabetes insipidus secondary to tuberculous meningoencephalitis with hypothalamic involvement extending to the hypophysis: a case report
}

\author{
Monique Freire Santana[1],[2], Guilherme Augusto Pivoto João[1], \\ Marcus Vinicius Guimarães de Lacerda ${ }^{[1]}$ and Luiz Carlos de Lima Ferreira ${ }^{[1],[3]}$
}

[1]. Fundação de Medicina Tropical Dr. Heitor Vieira Dourado, Manaus, AM, Brasil.

[2]. Departamento de Ensino e Pesquisa, Fundação Centro de Controle de Oncologia do Amazonas, Manaus, AM, Brasil. [3]. Departamento de Patologia e Medicina Legal, Universidade Federal do Amazonas, Manaus, AM, Brasil.

\begin{abstract}
The involvement of Mycobacterium tuberculosis in the central nervous system (CNS) is an uncommon and devastating manifestation of tuberculosis. We report a case of disseminated tuberculosis presenting as meningoencephalitis, hypothalamic involvement with extension to the hypophysis, and secondary insipidus diabetes diagnosed at autopsy.
\end{abstract}

Keywords: Tuberculosis. Meningoencephalitis. Hypothalamus. Hypophysitis. Insipidus diabetes.

\section{INTRODUCTION}

The involvement of Mycobacterium tuberculosis in the central nervous system (CNS) is a rare and devastating manifestation of tuberculosis (TB). The frequency of CNS TB is approximately $1 \%$ of all cases of $\mathrm{TB}$, with high mortality ${ }^{1}$. Clinical manifestations of CNS TB vary from meningitis, basilar arachnoiditis, tuberculoma, and encephalitis owing to devastating vasculopathy ${ }^{2}$. Tuberculous hypophysitis is a rare entity ${ }^{3}$, and secondary central insipidus diabetes due to sellar-suprasellar tuberculomas has been described elsewhere ${ }^{3}$. In 2015, the TB incidence rate in Brazil was 33.2 cases/100,000 inhabitants. The Amazonas State has the highest TB incidence rate, with 71.2 cases/100,000 inhabitants ${ }^{4}$. In 129 AIDS patients autopsied in the state of Amazonas, Souza et al. found that tuberculosis was a cause of death in $28 \%$ of cases $^{5}$. The association between TB and drug abuse, especially crack cocaine, is a growing concern ${ }^{6}$. We present a case report of meningoencephalitis associated with hypothalamic involvement with extension to the hypophysis that resulted in diabetes insipidus, secondary to disseminated infection with M. tuberculosis, with a fatal outcome diagnosed at autopsy.

\section{CASE REPORT}

The patient was a 31-year-old man who came from Manaus (capital of the Amazonas State, Western Brazilian Amazon);

Corresponding author: Monique Freire Santana.

e-mail: monique.freire20@gmail.com

Received 10 December 2017

Accepted 18 July 2018 he was an industrial worker, smoker, and user of illicit drugs (crack cocaine and marijuana) without previous contact with tuberculous patients. He sought medical care because of complaints of a headache, neck stiffness, behavioral changes, and otalgy on the left side; this had developed over eight days and was associated with nausea, postprandial vomiting, and mental confusion. The patient was admitted to the hospital. IV ceftriaxone and dexamethasone were promptly begun, and cerebral abscess of the temporal lobe was suspected. Fortyeight hours before hospital admission, a computed tomography (CT) scan was performed without alterations. Cerebrospinal fluid analysis disclosed mild lymphocytic pleocytosis with elevated protein levels. The patient developed a dry cough, otorrhea in the left ear, and periods of agitation; vancomycin was then added to the regimen. Given the clinical and laboratory suspicion of tuberculous meningoencephalitis, the COXCIP-4 scheme was started empirically. One day later, the patient was admitted to the intensive care unit with maintenance of the sensory level, respiratory distress with Kussmaul rhythm, seizures, hypotension, anisocoric pupils $\mathrm{L}>\mathrm{R}$ without reaction to light, and diffuse respiratory snoring. The next day the patient developed refractory hemodynamic instability, polyuria with six $\mathrm{mL} / \mathrm{kg}$ /hour urine output, and serious hypernatremia leading to death. The patient died within 20 days of the onset of symptoms. An autopsy was performed. The ectoscopy was unremarkable except for severe malnutrition. At the thoracic cavity overture, bilateral pleural-thoracic adherences were observed.

The lungs showed hemorrhagic areas at the base and micro-nodules with a millium appearance at the apical regions. On microscopy, the pulmonary parenchyma showed diffuse 
granulomas, some with caseous necrosis. The Ziehl-Neelsen stain showed a few acid-fast bacilli (AFB). In the abdominal cavity, the diaphragmatic surface had several white-yellowish micronodules, and on microscopic examination, they are represented by well-defined granulomas. The liver, spleen, bone marrow, kidneys, and adrenal glands were not altered in the macroscopic examination; however, all of these organs showed granulomatous involvement on microscopy. CNS examination showed diffuse edema with intense exudate in the optic chiasma region covering the brain stem, and many dots of hemorrhages at the cerebral surface. The basal meninges demonstrated thick, gelatinous subarachnoid exudate that filled the basal cisterns and diffuse leptomeningitis (Figure 1). Coronal sections of the cerebral hemispheres showed a necrotic lesion involving the left basal ganglia and the adjacent part of the claustrum, the hypothalamic region bilaterally, which was more accentuated to the left, and the inferior part of the right basal ganglia, extending to the junction of the claustrum with the superior temporal gyrus (Figure 2), granular exudate in the lateral ventricles, and hydrocephalus. Granulomatous ventriculitis with a few AFB on Ziehl-Neelsen stain was seen, as well as several fibrin thrombi with vasculitis, fibrinoid necrosis of the vessel walls, and an intense perivascular inflammatory infiltrate consisting of neutrophils, lymphocytes, and histiocytes (Figure 3). The necrotic lesions in the basal ganglia showed vascular neoformation and eosinophilic neurons with pyknotic nuclei, which are characteristic of cerebral infarction. The subarachnoid space was filled by an intense inflammatory infiltrate with neutrophils, lymphocytes, and histiocytes, with infiltration of the adjacent brain parenchyma. The hypothalamus and pituitary gland showed diffuse necrosis on gross pathology, with partial destruction of the pituitary parenchyma by an intense inflammatory infiltrate of lymphocytes and epithelioid cells (Figure 4). A Grocott stain was performed in all tissues with granulomatous involvement, which were negative for fungal structures.

\section{DISCUSSION}

CNS TB is uncommon and frequently fatal ${ }^{1}$. Hydrocephalus, vasculitis-causing infarction, and cranial neuropathies have been described as complications of tuberculous meningitis (TBM). Other manifestations are pachymeningitis, granulomatous basal meningitis, parenchymal tuberculosis (included parenchymal tuberculomas, tuberculous abscesses, miliary tuberculomas, focal tuberculous cerebritis, and encephalopathy), tuberculoma en plaque, tuberculous hypophysitis, involvement of the calvarium and the base of skull, orbital tuberculosis, tuberculous otitis media, temporal bone tuberculosis, and spinal tuberculosis ${ }^{7}$. Tuberculous ventriculitis is an uncommon disease, with only a few cases reported ${ }^{8}$. Small superficial tuberculomas are quite common in tuberculous leptomeningitis; however, large tuberculomas are rare in patients with leptomeningitis, whereas infarction may be present in $25 \%$. In these patients, predictors of a poor outcome are old age, high TBM grading, presence of infarction, and hydrocephalus'. In the present case, no tuberculoma was found; however, several infarction areas were present with extensive destruction of the parenchyma, which is associated with hydrocephalus.

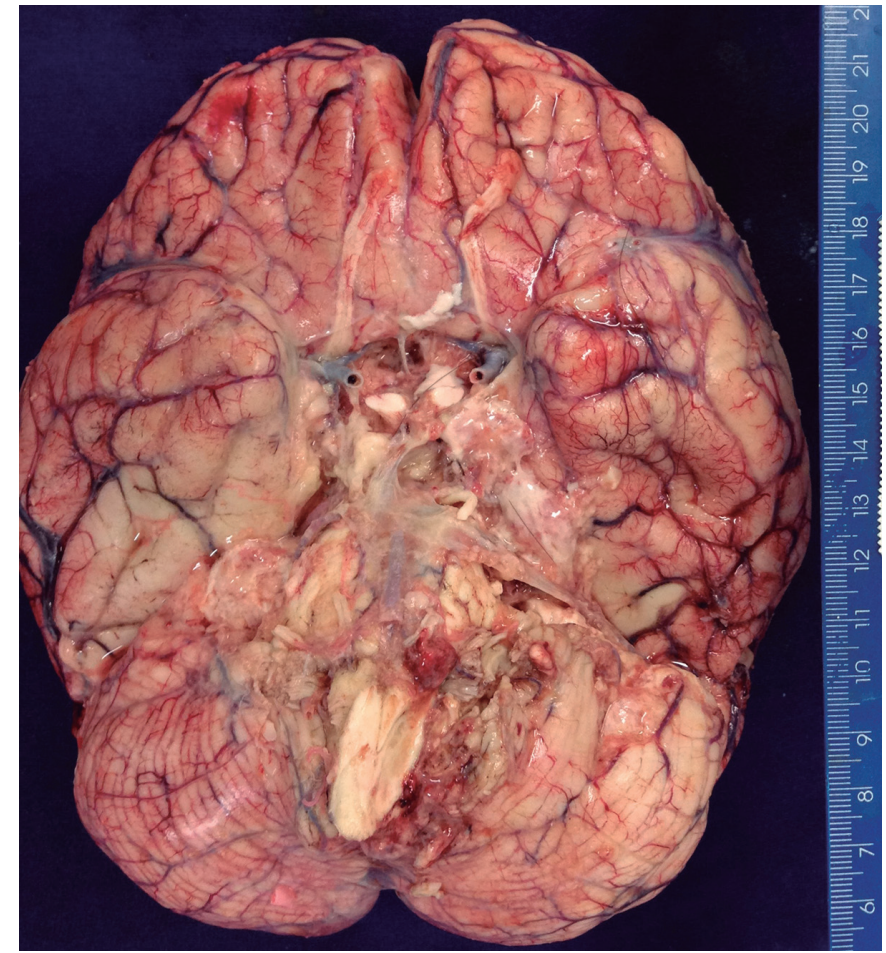

FIGURE 1: Gross pathology of brain: Thick, gelatinous subarachnoid exudate that fills the basal cisterns.

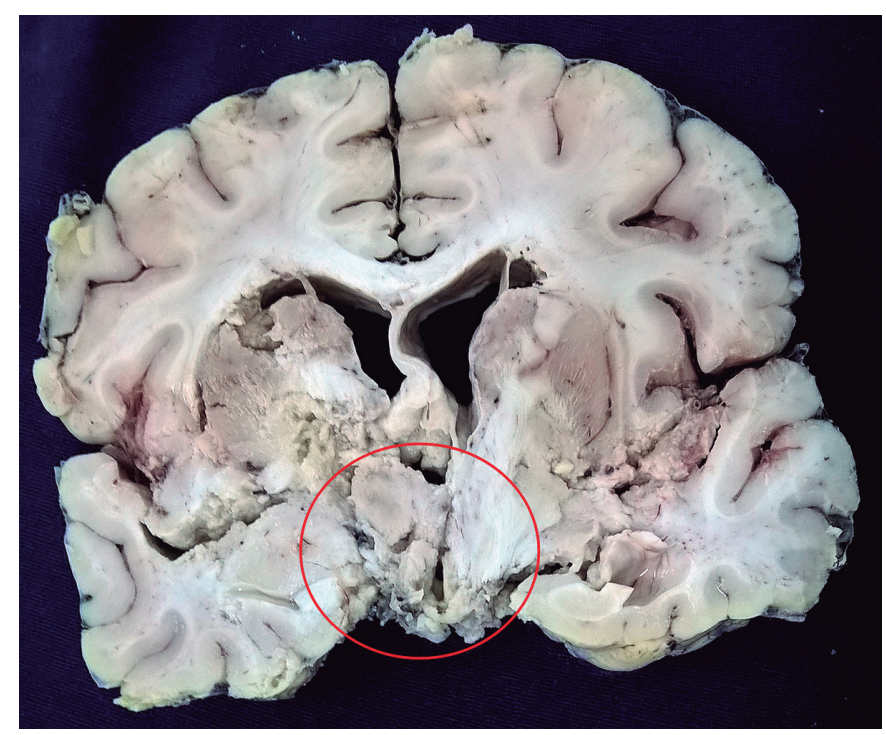

FIGURE 2: Cross-sections of brain: Extensive necrotic lesion involving the left basal ganglia and the claustrum, hypothalamus (circle), and the inferior part of the right basal ganglia.

In comparison to patients with noninflammatory ischemic stroke (IS), the most frequent infarction areas in TBM were described in the TB zone, which is supplied by the medial striate and thalamoperforating arteries. In patients with IS, the most commonly affected area was supplied by the lateral striate, anterior choroidal, and thalamogeniculate arteries; however, the opposite was observed in patients with $\mathrm{TBM}^{10}$.

TB cerebral vasculitis can be included in the differential diagnosis in complications of any case of tuberculous meningitis. 


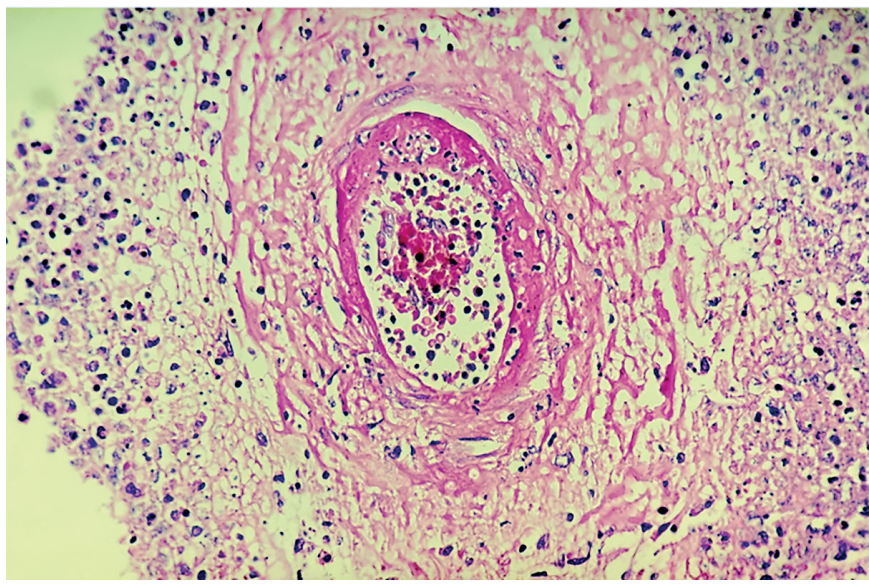

FIGURE 3: Tuberculous cerebral vasculitis: Vasculitis with fibrinoid necrosis (Hematoxylin \& Eosin, 200x).

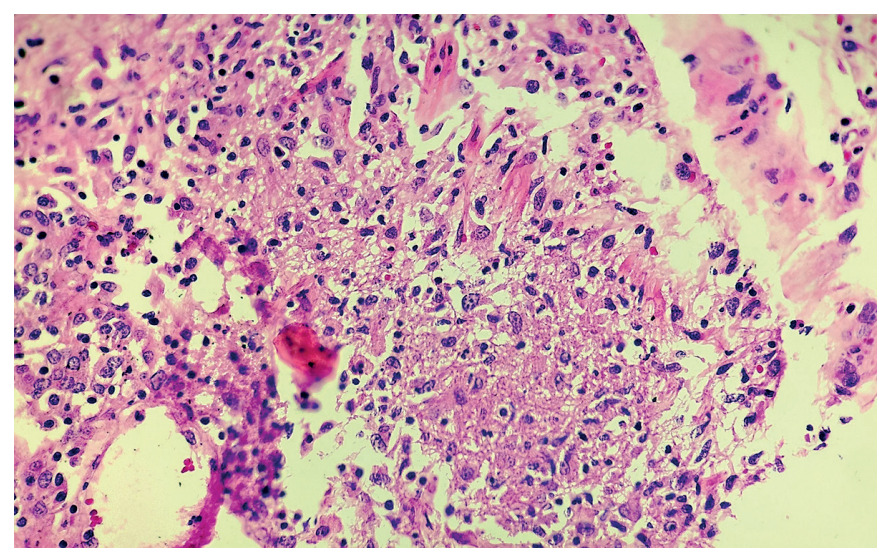

FIGURE 4: Tuberculous Hypophysitis: Parenchymal destruction by intense inflammatory infiltrated with lymphocytes and epithelioid cells (Hematoxylin \& Eosin, 400x).

In a 41-year-old woman with TBM, tuberculous vasculitis was seen on day 30 , with perforated infarction occurring around Willis' circle and debris located at the base of the skull, without evidence of the narrowing of an artery inside the brain. With supportive care and an increased dosage of prednisolone, her condition gradually improved without significant neurological disorder ${ }^{11}$. In this case, the patient presented with several cerebral infarctions as a complication of extensive vasculitis, probably leading to the poor prognosis. Pituitary TB is very rare. The most common presentation is chronic granulomatous inflammation associated with hypopituitarism ${ }^{12}$. The present patient died because of tuberculous meningoencephalitis, with extensive cerebral infarctions secondary to vasculitis and hypothalamic/pituitary involvement associated with diabetes insipidus. This case draws attention to the broad spectrum of involvement by M. tuberculosis in the CNS. In geographic areas with a high prevalence of tuberculosis, empirical treatment must be performed early because of the commonly fatal outcome.

\section{Ethical considerations}

The autopsy was performed after relatives' formal consent.

\section{Acknowledgments}

The authors thank the Fundação de Medicina Tropical Dr. Heitor Vieira Dourado and the Post-Graduate Program in Tropical Medicine, from Universidade do Estado do Amazonas, for the encouragement and support of scientific publications.

\section{Conflict of Interest}

The authors declare that there is no conflict of interest.

\section{REFERENCES}

1. Rock RB, Olin M, Baker CA, Molitor TW, Peterson PK. Central nervous system tuberculosis: Pathogenesis and clinical aspects. Clin Microbiol Rev. 2008;21(2):243-61.

2. Christie LJ, Loeffler AM, Honarmand S, Flood JM, Baxter R, Jacobson S, et al. Diagnostic challenges of central nervous system tuberculosis. Emerg Infect Dis. 2008;14(9):1473-5.

3. Bano S, Chaudhary V, Yadav S. Central Nervous System Tuberculosis. In: Bright P, editor. Neuroimaging - Clinical Applications [Internet]. INTECH Open Access Publisher; 2012. Available from: http://www.intechopen.com/books/neuroimagingclinical- applications

4. Satyarthee GD, Mahapatra AK. Diabetes insipidus in sellarsuprasellar tuberculoma. J Clin Neurosci. 2003;10(4):497-9.

5. Brasil M da S. Série histórica da Taxa de Incidência de Tuberculose. Brasil, Regiões e Unidades Federadas de residência por ano de diagnóstico (1990 a 2015). 2015.

6. Souza SLS de, Feitoza PVS, Araújo JR de, Andrade RV de, Ferreira LCDL. Causas de óbito em pacientes com síndrome da imunodeficiência adquirida, necropsiados na Fundação de Medicina Tropical do Amazonas. Rev Soc Bras Med Trop. 2008;41(3):247-51.

7. Story A, Bothamley G, Hayward A. Crack cocaine and infectious tuberculosis. Emerg Infect Dis. 2008;14(9):1466-9.

8. Kumar S, Kumar R, Radotra BD, Singh M. Tubercular ventriculitis: An uncommon entity. Indian J Pediatr. 2014;81(6):608-10.

9. Wasay M, Farooq S, Khowaja ZA, Bawa ZA, Ali SM, Awan S, et al. Cerebral infarction and tuberculoma in central nervous system tuberculosis: frequency and prognostic implications. J Neurol Neurosurg Psychiatry. 2014;85(11):1260-4.

10. Hsieh FY, Chia LG, Shen WC. Locations of cerebral infarctions in tuberculous meningitis. Neuroradiology. 1992;34(3):197-9.

11. Chen CH, Chen YM, Chen WL, Yang YJ, Lai CH. Late-onset cerebral vasculitis with tuberculous meningitis: A case report. J Intern Med Taiwan. 2014;25(5):362-70.

12. Khan K, Drake W, Akker S, White V. P50 Pituitary Granulomata TB or Not TB. Thorax. 2012;67(Suppl 2):A85-A85. 